\title{
SEXTANT X-ray Pulsar Navigation Demonstration: Additional On-Orbit Results ${ }^{* \dagger}$
}

\author{
Luke M. B. Winternitz $\$$, Jason W. Mitchell§, Munther A. Hassouneh», \\ Samuel R. Price§ Sean R. Semper§, Wayne H. Yu, Paul S. Ray, Michael T. Wolff,

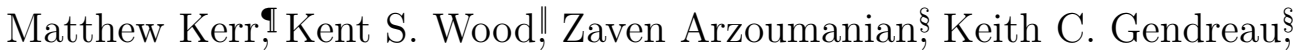 \\ Lucas Guillemot,* Ismaël Cognard** \\ Paul Demorest,$^{\dagger \dagger}$ Ben Stappers, ${ }^{\ddagger \ddagger}$ Andrew Lyne ${ }^{\ddagger \ddagger}$
}

\begin{abstract}
The Station Explorer for X-ray Timing and Navigation Technology (SEXTANT) is a technology demonstration enhancement to the Neutron-star Interior Composition Explorer (NICER) mission, a NASA Astrophysics Explorer Mission of Opportunity to the International Space Station, launched in June of 2017. In late 2017, SEXTANT successfully completed a first demonstration of in-space and autonomous $\mathrm{X}$-ray pulsar navigation (XNAV). This form of navigation relies on processing faint signals from millisecond pulsars - rapidly rotating neutron stars that appear to pulsate in the X-ray band - and could potentially provide a GPS-like navigation capability applicable throughout the solar-system and beyond. In this work, we briefly review prior SEXTANT results and then present new results focusing on: making use of the high-flux but rotationally unstable Crab pulsar, and using XNAV to estimate position, velocity, and time in the presence of an imperfect local clock.
\end{abstract}

\section{Background}

The Station Explorer for X-ray Timing and Navigation Technology (SEXTANT) mission is a technology demonstration enhancement to the Neutron-star Interior Composition Explorer (NICER) mission aimed at enabling autonomous navigation for future missions operating far from Earthbased navigational resources $[1,7,10,11]$. On June $3^{\text {rd }}$ of 2017 , NICER/SEXTANT launched to the International Space Station (ISS) to begin an 18-month baseline mission. NICER is an X-ray

\footnotetext{
${ }^{*}$ This material is declared a work of the U.S. Government and is not subject to copyright protection in the United States. All other rights reserved.

${ }^{\dagger}$ This work is funded by NASA Space Technology Mission Directorate, Game Changing Development Program

${ }^{\ddagger}$ luke.b.winternitz@nasa.gov@nasa.gov

$\S$ NASA Goddard Space Flight Center, Greenbelt, MD 20771, USA

${ }^{\mathbb{I}}$ U.S. Naval Research Laboratory, Washington, DC 20375, USA

" Praxis Inc., resident at Naval Research Laboratory, Washington, DC 20375, USA

** Laboratoire de Physique et Chimie de l'Environnement et de l'Espace, Université d'Orléans/CNRS, F-45071 Orléans Cedex 02, FRANCE and Station de Radioastronomie de Nançay, Observatoire de Paris, CNRS/INSU, F18330 Nançay, FRANCE

${ }^{\dagger \dagger}$ National Radio Astronomy Observatory, Socorro, NM 87801, USA

‡ł Jodrell Bank Centre for Astrophysics, The University of Manchester, Manchester M13 9PL, UK
} 
astrophysics mission with the goal of characterizing the emission and rotation properties of pulsars to constrain the dense matter equation of state, while SEXTANT aims to exploit their predictable pulse timing information for autonomous navigation, a process often referred to as X-ray Pulsar Navigation (XNAV).

The primary performance goal of the SEXTANT mission is to achieve real-time, onboard, navigation position accuracy of $10 \mathrm{~km}$ worst-direction, using up to two weeks of dedicated navigation-focused Millisecond Pulsar (MSP) observations. This uncertainty is calculated as $<17.3 \mathrm{~km}$ Root Sum Square (RSS) error compared to the onboard GPS solution, whose accuracy is several orders of magnitude better. The onboard navigation demonstration starts with an intentionally-degraded orbit position and velocity, based on data provided by NICER's Global Positioning System (GPS) receiver, and then must maintain orbit knowledge by processing only X-ray emitting MSP observations. Prior to the flight demonstration, NICER made a series of observations of each SEXTANT pulsar to calibrate pulsar timing models (pulsar rotational ephemerides and absolute X-ray phase) for use in the experiment. The rotational ephemerides were derived from ground-based radio measurements in the form of arrival times from ongoing long-term pulsar monitoring campaigns. The absolute X-ray phase of the pulses was then determined from early NICER observations. The resulting timing models were then used for the generation of Time-of-Arrival (TOA) predictions for the duration of the experiment. During the calibration campaign, several successful ground experiments were conducted by replaying flight telemetry into the X-ray Pulsar Navigation Flight Software (XFSW) and/or playing the events back through the SEXTANT XNAV Laboratory Testbed (XLT) end-to-end simulation, see [8]. During the replay of flight telemetry from 2017 Day-of-Year (DOY) 259.5 (September 16 ${ }^{\text {th }}$ at 12:00 UTC) to DOY 264.5 (September 21 $1^{\text {th }}$ at 12:00 UTC), a ground experiment's navigational errors successfully met the SEXTANT performance goal. These early results provided a critical step in understanding the onboard performance for the actual flight demonstration.

The 2017 flight navigation experiment, conducted between November 10 ${ }^{\text {th }}$ (DOY 314) through the $12^{\text {th }}$ (DOY 316), met its primary goal by achieving autonomous XNAV navigation performance better than $10 \mathrm{~km}$, worst direction, onboard and in real-time. Mitchell et al. [8] provide a discussion the details of the flight experiment results, including predictive Monte-Carlo simulation results and XFSW initialization parameters.

In this work, a companion to [8], we report on additional SEXTANT results that focus on two fronts. First, we discuss the use of the high-flux, but rotationally-unstable Crab pulsar for XNAV, describing calibration of the Crab pulsar models, and present a successful navigation result that relies heavily on observations of the Crab pulsar. Second, we present an enhancement to the SEXTANT algorithms to enable XNAV with time estimation in the presence of an imperfect local clock, and present an experimental result reprocessing NICER/SEXTANT flight telemetry.

\section{The Crab Pulsar}

The Crab pulsar, also known as PSR B0531+21, and its radio timing properties have been extensively studied for over 45 years [6]. In the X-ray band, the pulsed emission from the Crab pulsar is three orders of magnitude brighter than any of the SEXTANT MSP sources and has a pulse profile with a relatively narrow main peak of only $250 \mu \mathrm{s}$ in width. As a result, X-ray TOAs suitable for navigation applications can be measured in very short observations, e.g., a high quality measurement with $10 \mu \mathrm{s}$ statistical precision can be obtained in only about $600 \mathrm{~s}$ with NICER, and observations of only a few 10s of seconds can provide useful information, as well. This makes the 


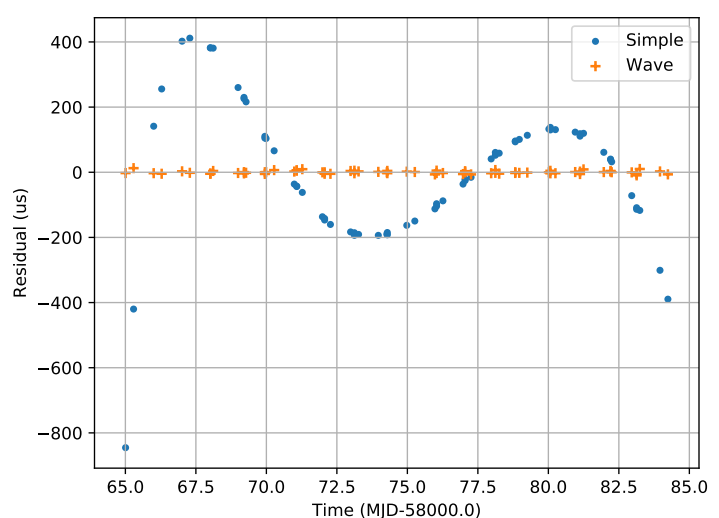

Figure 1: Timing residuals from Jodrell Bank TOAs for the Crab pulsar using two timing models. The Simple model has frequency derivatives only up to $\ddot{f}$, while the WAVE (FITWAVE) model adds harmonically-related sinusoids to model the timing noise.

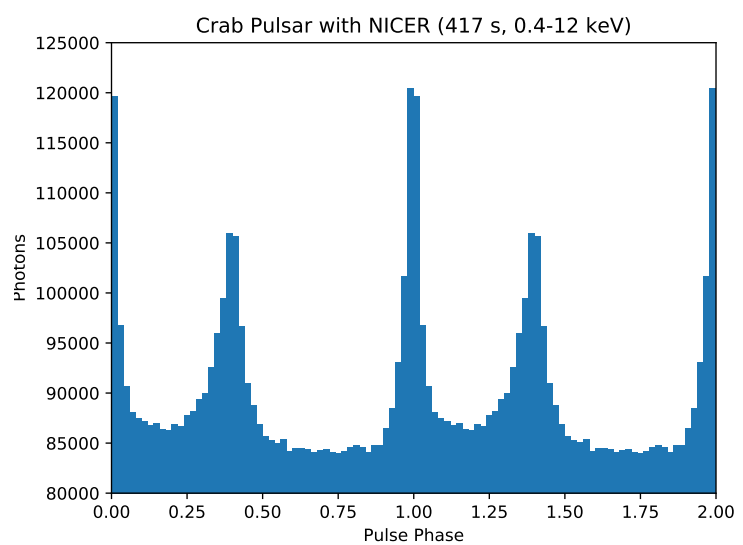

Figure 2: Crab pulsar profile from 417 seconds of NICER data, in the $0.4-12 \mathrm{keV}$ band.

Crab pulsar an appealing source for XNAV either with a small detector system or in a situation where very frequent measurements are required to keep the navigation solution accurate. Unfortunately, the strong timing noise in the Crab pulsar prevent the ground-based timing solution from being extrapolated to make accurate predictions more than a few days from the last measurement [9]. But, with sufficiently frequent updates of the predictive timing model, the Crab pulsar can be used for onboard navigation and related applications. This section summarizes the key concepts and techniques required for processing observations from the Crab pulsar.

\section{A. NICER Crab Pulsar Calibration}

To use the Crab pulsar in the navigation processing, we need both a spindown model and an absolute time reference. In the following subsections, we describe how the spin ephemeris was derived from radio monitoring observations, then how the absolute time and pulse profile template were derived from NICER X-ray observations.

\section{Radio Observations}

The Crab pulsar is observed daily at the Jodrell Bank Observatory and we used these observations to produce a timing model for our navigation demonstration. We acquired TOAs from 2017 November $8^{\text {th }}$ through the $27^{\text {th }}$ (DOYs 312-331). To illustrate the magnitude of the timing noise, we first fitted the arrival times to a simple model consisting only of position, dispersion measure and a spindown model consisting of frequency, frequency derivative and frequency second derivative. The residuals to this model fit are shown in Fig. 1, where the pulses wander around the simple model by several hundred microseconds. Using this model would result in systematic errors in the navigation measurements of over $100 \mathrm{~km}$. Instead, we added five harmonically-related sinusoidal terms to the model account for the timing noise, see the FITWAVES procedure as described by [2]. The residuals to this fit are nearly white and have an Root Mean Square (RMS) error of only 3.8 us, which is 
comparable to the uncertainties on the individual arrival time measurements. This is the model we used for the navigation exercise. It is important to note that this allows the high precision measurements of the Crab pulsar to be used for navigation in a post-processed mode of operation, but is not representative of a situation where a navigation solution must be derived in real time, based on an extrapolated timing model as is done with the MSPs.

\section{NICER Data Processing}

The radio timing model predicts the pulse phase evolution accurately, but for navigation, we require a precise absolute arrival time in the NICER X-ray band, based on a template profile that defines the fiducial point for X-ray phase measurements.

We extracted 417 seconds of NICER data on the Crab pulsar from November $11^{\text {th }} 2017$ (ObservationID 1013010111) between $0.4 \mathrm{keV}$ and $12 \mathrm{keV}$, applying standard cuts by energy band to remove non-photon events. Figure 2 shows the pulse profile of the Crab pulsar derived from that observation. The pulsed signal in the $0.4-12 \mathrm{keV}$ band has a phase-averaged rate of 909 counts/s on top of an unpulsed background rate of 10,022 counts/s that is predominately from the surrounding nebula. The NICER instrumental background in this band is insignificant at less than 0.5 counts/s.

We model the pulse profile shape as the sum of two 2-sided Lorentzians plus a broader Gaussian. We define phase 0.0 as the peak of the first narrow Lorentzian. The Full Width Half Maximum (FWHM) of this peak is $252 \mathrm{\mu s}$. We use a binned version of this analytic profile shape as the template for measuring pulse arrival times.

Using this template, we measured the absolute phase using the photon_toa.py code from PINT [5] to extract a TOA from a single NICER observation. This TOA corresponds to the fiducial point on the template profile. We inserted that time into the timing model as the definition of phase zero for the pulsar, as defined by the TZRMJD parameter ${ }^{1}$. This calibrated timing model was then used for the Crab pulsar navigation experiments described below.

\section{SEXTANT Measurement Model Extension for Clock and Measurement Bias Estimation}

In the SEXTANT navigation filter, pulse phase and, optionally, frequency measurements are blended with models of the spacecraft dynamics to update an estimate of the spacecraft state. Here, we present a simple extension of the SEXTANT measurement model presented in [10] that allows the filter to estimate a local clock bias and rate, and a per-pulsar measurement bias. Later, we present results obtained by reprocessing NICER/SEXTANT ground telemetry that has been perturbed by simulated clock errors and show that the filter is able to recover accurate estimates of these errors. While experiments with per-pulsar measurement biases are not presented in this work, it is convenient to present the measurement model extension for both error types together, as we believe the measurement model may have value for future experiments especially involving the Crab pulsar.

Let the spacecraft position, velocity, clock bias, clock rate, and a constant measurement bias at

\footnotetext{
${ }^{1}$ The fiducial point on the radio profile arrives at site TZRSITE at frequency TZRFRQ at the moment TZRMJD in Modified Julian Date (MJD).
} 
time $t$ be given by $\mathbf{x}(t), \mathbf{v}(t), b(t), r(t)$, and $\delta \phi$, respectively, and let $\hat{\mathbf{n}}$ be the unit direction to the pulsar and $c$ the speed-of-light in vacuum. At time $t$, the pulsar pulse phase $\phi(t)$ observed at the spacecraft is related to the phase $\phi_{0}(t)$ at the reference observatory by ${ }^{2}$

$$
\phi(t)=\phi_{0}(t-b(t) / c+\hat{\mathbf{n}} \cdot \mathbf{x}(t) / c)+\delta \phi
$$

where the reference observatory is taken to be at the geocenter for SEXTANT, making relative parallax and solar system Shapiro delays, etc., negligible for our purposes.

The frequency measurement equation is obtained by differentiating Eq. (1) with respect to $t$, leading to

$$
\dot{\phi}(t)=\frac{d}{d t} \phi(t)=\dot{\phi}_{0}(t-b(t) / c+\hat{\mathbf{n}} \cdot \mathbf{x}(t) / c)(1-r(t) / c+\hat{\mathbf{n}} \cdot \mathbf{v}(t) / c)
$$

where $\mathbf{v}:=\dot{\mathbf{x}}$ and $r:=\dot{b}$. The filter also requires the first order partial derivatives of Eqs. (1) and (2) with respect to the estimated state, which are given by

$$
\frac{\partial \mathbf{y}}{\partial \mathbf{z}}=\frac{1}{c}\left(\begin{array}{ccccc}
f(t) \hat{\mathbf{n}}^{\top} & \mathbf{0}^{\top} & -f(t) & 0 & c \\
\beta(t) a(t) \hat{\mathbf{n}}^{\top} & f(t) \hat{\mathbf{n}}^{\top} & -\beta(t) a(t) & -f(t) & 0
\end{array}\right),
$$

with $\mathbf{y}=(\phi, \dot{\phi})^{\top}, \mathbf{z}=(\mathbf{x}, \dot{\mathbf{x}}, b, r, \delta \phi)$, and

$$
\begin{aligned}
f(t) & :=\dot{\phi}_{0}(t-b(t) / c+\hat{\mathbf{n}} \cdot \mathbf{x}(t) / c), \\
a(t) & :=\ddot{\phi}_{0}(t-b(t) / c+\hat{\mathbf{n}} \cdot \mathbf{x}(t) / c), \\
\beta(t) & :=(1-r(t) / c+\hat{\mathbf{n}} \cdot \mathbf{v}(t) / c) .
\end{aligned}
$$

\section{Navigation Experiments}

The SEXTANT team has developed an extensive suite of XNAV simulation tools whose capabilities are described in detail in previous publications $[8,10,11]$. These capabilities include the ability to replay NICER/SEXTANT flight telemetry through the SEXTANT XFSW application running on a ground computer. This mode is used for the Crab pulsar experiment described below. The timing experiment, also described below, uses a second mode of simulation, where telemetry is simulated or played back through the SEXTANT end-to-end simulation, which runs the XFSW as a component, but includes additional Monte-Carlo capabilities.

These experiments make use of the same MSP calibrations described in [8]. Thus, we only describe the new calibration developed for the Crab pulsar below.

\footnotetext{
${ }^{2}$ Note there is a sign error in [10], in the corresponding Eq. (14), in front of the propagation delay term. Here, we present the correct form used in the SEXTANT flight software.
} 


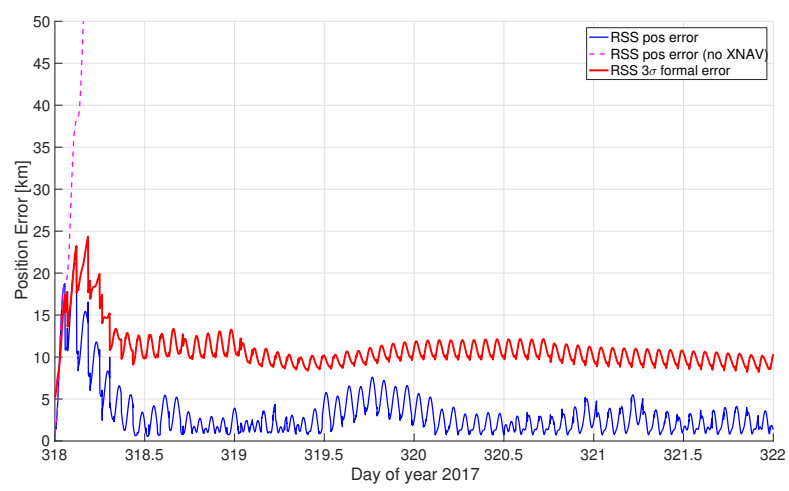

(a) Result from Crab pulsar ground experiment, showing RSS position errors (XNAV vs. GPS solution) and $3 \sigma$ RSS filter root-covariance. The dashed line gives the RSS error obtained by simply propagating the initial degraded state without processing XNAV measurements, which rapidly diverges.

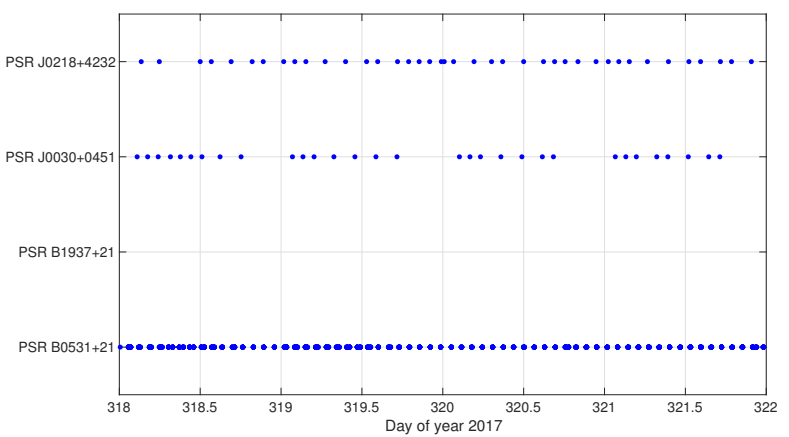

(b) Crab pulsar experiment observation schedule.

Figure 3: Crab pulsar ground experiment results.

\section{A. Crab Pulsar Ground Experiments}

In this section, we describe results from a SEXTANT ground experiment where the Crab pulsar (PSR B0531+21) was processed using a binned approach to phase estimation as described in [11]. In this experiment, X-ray event data from NICER/SEXTANT flight telemetry were collected over a period from 2017 DOYs 318-322 and replayed through the XFSW application on the ground. This period includes high-cadence Crab pulsar observations along with additional observations of the MSPs PSR J0030+0451 and PSR J0218+4232. The observation schedule is shown in Fig. 3b. For this experiment, the timing model for the Crab pulsar was developed using radio data spanning 2017 DOYs 315-335 as described in Section II, enveloping the experiment period. Thus, this experiment represents the first SEXTANT demonstration of a post-processed navigation capability using the Crab pulsar.

Figure 3a shows the position RSS errors achieved by the SEXTANT navigation system after seeding with an degraded GPS solution with a $(500 \mathrm{~m}, 500 \mathrm{~m}, 500 \mathrm{~m}, 0.5 \mathrm{~m} / \mathrm{s}, 0.5 \mathrm{~m} / \mathrm{s}, 0.5 \mathrm{~m} / \mathrm{s})$ Earthcentered, Earth-fixed error (see [8] for discussion) and processing Crab pulsar phase measurements using very short $10 \mathrm{~s}$ observations along with a smaller number of phase and frequency measurements from two other MSPs. The filter converges to a steady state error below the $10 \mathrm{~km}$ RSS meeting the SEXTANT performance goal. Further investigation shows this error is primarily in-track, as is typical.

This demonstrates that Crab pulsar-based XNAV can support strong performance levels, providing benefit to missions needing high-cadence observations, e.g., high-dynamics scenarios or where the $\mathrm{X}$-ray timing detector is of smaller size, as compared to NICER.

\section{B. Time Estimation}

In this section, we describe results from a SEXTANT ground navigation experiment that investigates estimating the spacecraft position, velocity, as well as local clock bias and rate states. To 


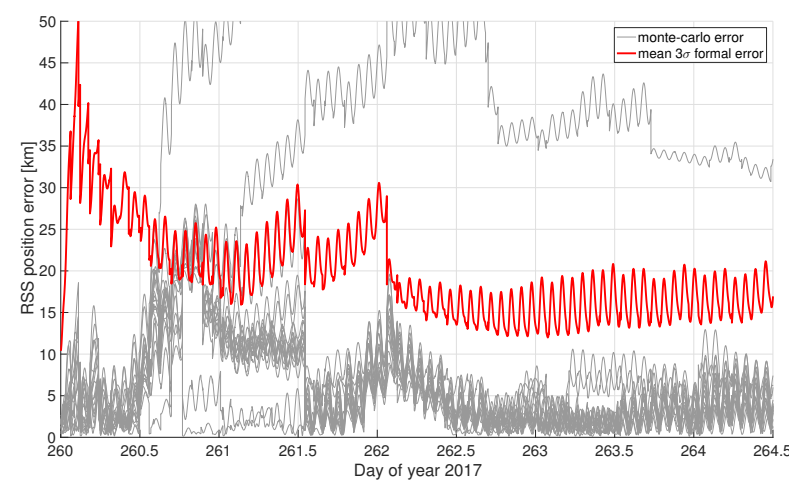

(a) XLT end to end simulation Monte-Carlo RSS position errors and mean $3 \sigma$ RSS filter root-covariance for the clock bias and rate estimation ground experiment scenario.

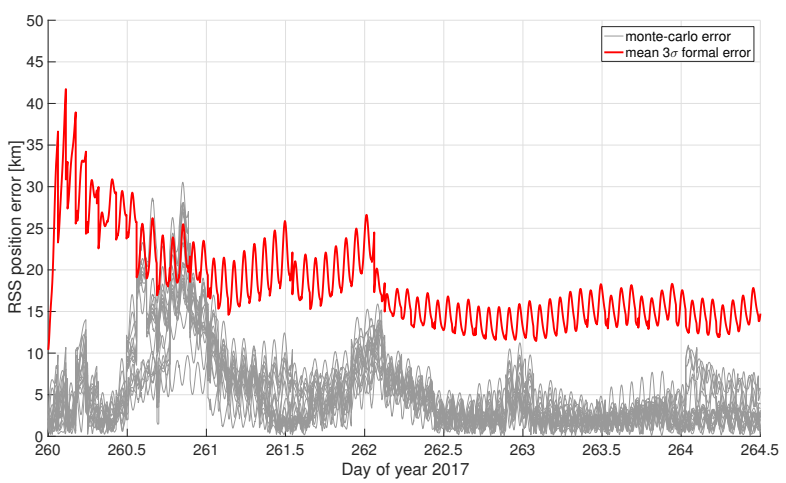

(b) XLT end to end simulation Monte-Carlo RSS position errors and mean $3 \sigma$ RSS filter root-covariance for the time-bias experiment scenario with clock errors turned off.

Figure 4: Results from clock estimation experiment.

support this experiment, we simulated random clock errors from a realistic model of an ultra-stable crystal oscillator and added these errors to the GPS timestamps of the detected X-ray events provided by NICER. We then replayed this perturbed flight telemetry through the XLT end-to-end simulation, which runs the XFSW as a component, and looked at navigation and time estimation performance using the augmented navigation filter model described in Section III.

To simulate the clock errors, we used a thrice-integrated Gaussian noise model with $q$-parameters, i.e., intensities of the bias, drift and acceleration driving noises, set to $1.2 \times 10^{-22} \mathrm{~s}^{2} / \mathrm{s}, 1.5 \times 10^{-26} \mathrm{~s}^{2} / \mathrm{s}^{3}$, and $1 \times 10^{-38} \mathrm{~s}^{2} / \mathrm{s}^{5}$, respectively, which were derived from specifications of a real-world spacequalified, moderate cost, ultra-stable crystal oscillator using the approach described in [3]. The initial bias and rate were drawn randomly from zero-mean Gaussian distributions with standarddeviations of $100 \mu \mathrm{s}$ and $1 \times 10^{-8} \mathrm{~s} / \mathrm{s}$, respectively, and the initial clock acceleration was set to zero. The simulated clock bias trajectories are shown in Fig. 5b in units of km, along with a typical clock rate trajectory in $\mathrm{m} / \mathrm{s}$.

As in [8], we focused on observations over the period 2017 DOYs 260-264.5. This period was preplanned as an ad-hoc ground calibration period for the SEXTANT team, during which many SEXTANT observation requests were incorporated into the operational schedule. In particular, a high-density of observations from the excellent XNAV pulsar B1937+21 were included during this period. A plot of the observation schedule for this period can be found in [8].

We ran 20 Monte-Carlo cases where we randomized the filter's initial state estimate with Gaussian errors whose $1 \sigma$ root-variance was set to $(1 \mathrm{~km}, 1 \mathrm{~km}, 1 \mathrm{~km}, 1 \mathrm{~m} / \mathrm{s}, 1 \mathrm{~m} / \mathrm{s}, 1 \mathrm{~m} / \mathrm{s})$ and with offdiagonal orbital correlations of -0.95 for radial position with in-track velocity and in-track position with radial velocity. The initial clock bias and rate state estimates were set to zero. We disabled the pulsar frequency measurement for this experiment for empirical purposes. Then, for comparison purposes, in order to see the impact of the addition and estimation of the clock errors, we ran an additional 20 cases with the clock errors set to zero and retuned the filter to ignore the clock states.

The result shown in Fig. 4a gives position RSS errors (XNAV vs. GPS solution) for each of the 20 cases overlaid, and (mean across runs) $3 \sigma$ RSS filter root-covariance, while Fig. 5a shows the clock bias and rate estimation errors. Most of the cases ( $90 \%$ here) are able to accurately estimate the 

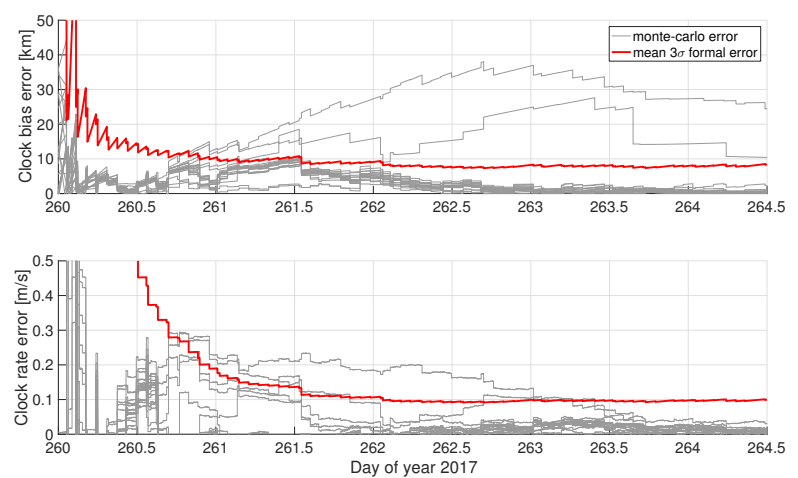

(a) Ground experiment clock bias and rate errors (estimated vs. truth sim) and $3 \sigma$ RSS filter rootcovariance.

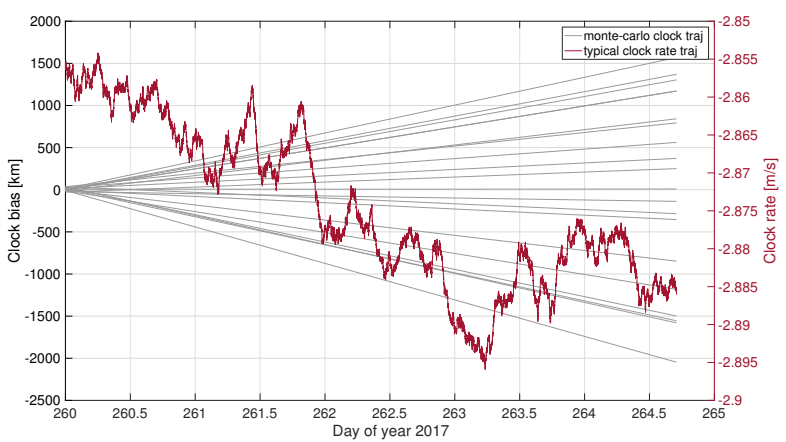

(b) Clock bias and a typical clock rate error trajectory used to perturb the X-ray event timestamps in the time estimation experiment.

Figure 5: Clock error estimation and trajectories from the experiment.

clock error states in addition to position (and velocity, not presented). Position estimation errors for the case with no clock errors are shown in Fig. 4b. Comparing this to Fig. 4a, we can see that, at least for this specific scenario, there is, perhaps not unexpectedly, some impact to the clock errors: there may be a small degradation in steady state position estimation error, and more obviously, an apparent increased chance of divergence (two divergent cases can be seen in Fig. 4a) when clock errors are present, as compared to when they are not. Nonetheless, the result is a positive one that demonstrates that position, velocity, and time estimation is possible with XNAV even with a relatively low-cost master spacecraft clock.

\section{Conclusion}

This paper serves as a complement to [8] and provides additional results from the Station Explorer for X-ray Timing and Navigation Technology (SEXTANT) demonstration. First we demonstrate post-processed navigation using the unique high-flux Crab pulsar, which has the great advantage of allowing for very short stare times of only 10s of seconds. Previous SEXTANT results have demonstrated XNAV position and velocity estimation, while taking advantage of the highly accurate timestamps from the NICER GPS receiver. In this paper, we present results from a ground simulation where we generated realistic clock errors from a model of an ultra-stable crystal oscillator, not an atomic clock, and show that the clock errors can be estimated accurately without major impact to position and velocity estimation performance. We also present an extended filter measurement model that enables this estimation.

For future work, SEXTANT is planning additional navigation experiments later in 2018, where timing models will be generated from NICER-only data. In addition, we will continue to execute ground and opportunistic flight experiments, and explore enhanced algorithms. Finally, we will continue infusion activities for future applications of XNAV.

\section{Acknowledgment}

We would like to acknowledge the NANOGrav Timing Working group for making their pulsar timing data available for this work. Contributors the the NANOGrav 11-year data set include: 
Zaven Arzoumanian, Kathryn Crowter, Megan DeCesar, Paul Demorest, Timothy Dolch, Justin Ellis, Elizabeth Ferrara, Robert Ferdman, Emmanuel Fonseca, Peter Gentile, Glenn Jones, Megan Jones, Michael Lam, Lina Levin, Duncan Lorimer, Ryan Lynch, Maura McLaughlin, Cherry Ng, David Nice, Timothy Pennucci, Scott Ransom, Paul Ray, Renee Spiewak, Ingrid Stairs, Kevin Stovall, Joseph Swiggum, and Weiwei Zhu.

Arzoumanian, Demorest, and Ray are members of the NANOGrav Physics Frontiers Center, which is supported by NSF award 1430284.

The Nançay Radio Observatory is operated by the Paris Observatory, associated with the French Centre National de la Recherche Scientifique (CNRS).

The Parkes Pulsar Timing Array data was taken with the Parkes Telescope, which is part of the Australia Telescope National Facility and is funded by the Australian Government for operation as a National Facility managed by the Commonwealth Scientific and Industrial Research Organisation (CSIRO).

The Jodrell Bank Observations were acquired with support from Cees Bassa and Mitch Mickaliger. Pulsar research at Jodrell Bank Center for Astrophysics is supported by a Consolidated Grant from the UK Science and Technology Facilities Council (STFC).

The Naval Research Laboratory collaboration with SEXTANT is supported by NASA Space Technology Mission Directorate, Game Changing Development Program.

Software Routines from the IAU SOFA Collection were used. Copyright (C) International Astronomical Union Standards of Fundamental Astronomy [4].

\section{References}

[1] Z. Arzoumanian, K. C. Gendreau, C. L. Baker, T. Cazeau, P. Hestnes, J. W. Kellogg, S. J. Kenyon, R. P. Kozon, K.-C. Liu, S. S. Manthripragada, C. B. Markwardt, A. L. Mitchell, J. W. Mitchell, C. A. Monroe, T. Okajima, S. E. Pollard, D. F. Powers, B. J. Savadkin, L. B. Winternitz, P. T. Chen, M. R. Wright, R. Foster, G. Prigozhin, R. Remillard, and J. Doty. The Neutron-star Interior Composition Explorer (NICER): Mission Definition. In Astronomical Telescopes and Instrumentation, volume 9144 of Proc. SPIE, pages 914420914420-9. International Society for Optics and Photonics, 2014. doi: 10.1117/12.2056811. URL http://dx.doi.org/10.1117/12.2056811.

[2] G. Hobbs, A. G. Lyne, M. Kramer, C. E. Martin, and C. Jordan. Long-term timing observations of 374 pulsars. MNRAS, 353:1311-1344, Oct. 2004. doi: 10.1111/j.1365-2966.2004.08157.x.

[3] S. T. Hutsell. Relating the hadamard variance to MCS kalman filter clock estimation. In Proceedsing of the 27th Annual Precise Time and Time Interval Applications and Planning Meeting, 1996.

[4] IAU SOFA Board. International Astronomical Union (IAU) Standards Of Fundamental Astronomy (SOFA) Software Collection. http://www.iausofa.org, 2017. URL http: //www. iausofa.org. 
[5] J. Luo et al. PINT: A Modern Software Package for Pulsar Timing, 2018. URL https: //github.com/nanograv/PINT. (in preparation).

[6] A. G. Lyne, C. A. Jordan, F. Graham-Smith, C. M. Espinoza, B. W. Stappers, and P. Weltevrede. 45 years of rotation of the crab pulsar. Monthly Notices of the Royal Astronomical Society, 446(1):857-864, 2015. doi: 10.1093/mnras/stu2118. URL http://dx.doi.org/10. $1093 / \mathrm{mnras} / \mathrm{stu} 2118$.

[7] J. W. Mitchell, M. A. Hassouneh, L. M. Winternitz, J. E. Valdez, S. R. Price, S. R. Semper, W. H. Yu, Z. Arzoumanian, P. S. Ray, K. S. Wood, R. J. Litchford, and K. C. Gendreau. SEXTANT - Station Explorer for X-ray Timing and Navigation Technology. In AIAA Guidance, Navigation, and Control Conference, AIAA SciTech. American Institute of Aeronautics and Astronautics, Jan. 2015. URL http://dx.doi.org/10.2514/6.2015-0865.

[8] J. W. Mitchell, L. B. Winternitz, M. A. Hassouneh, S. R. Price, S. R. Semper, W. H. Yu, P. S. Ray, M. T. Wolff, M. Kerr, K. S. Wood, Z. Arzoumanian, K. C. Gendreau, L. Guillemot, I. Cognard, and P. Demorest. SEXTANT X-Ray Pulsar Navigation Demonstration: Initial On-Orbit Results. In 41st Annual Guidance and Control Conference. American Astronautical Society, Feb. 2018.

[9] P. S. Ray, K. S. Wood, and M. T. Wolff. Characterization of pulsar sources for x-ray navigation. In W. Becker, editor, Proceedings of the 593. WE-Heraeus Seminar on Autonomous Spacecraft Navigation, 2018. in press, arXiv:1711.08507.

[10] L. Winternitz, M. Hassouneh, J. Mitchell, J. Valdez, S. Price, S. Semper, W. Yu, P. Ray, K. Wood, Z. Arzoumanian, and K. Gendreau. X-ray pulsar navigation algorithms and testbed for SEXTANT. In Aerospace Conference, 2015 IEEE. IEEE, March 2015. doi: 10.1109/AERO. 2015.7118936. URL http://dx.doi.org/10.1109/AERO.2015.7118936.

[11] L. Winternitz, J. Mitchell, M. Hassouneh, J. Valdez, S. Price, S. Semper, W. Yu, P. Ray, K. Wood, Z. Arzoumanian, and K. Gendreau. SEXTANT X-ray Pulsar Navigation Demonstration: Flight System and Test Results. In Aerospace Conference, 2016 IEEE. IEEE, March 2016.

10 of 10 